\title{
On the impossibility of fair risk allocation*
}

\author{
Péter Csóka $\quad$ Miklós Pintér ${ }^{\ddagger}$
}

July 29, 2015

\begin{abstract}
Allocating risk properly to subunits is crucial for performance evaluation and internal capital allocation of portfolios held by banks, insurance companies, investment funds and other entities subject to financial risk. We show that by using coherent measures of risk it is impossible to allocate risk satisfying simultaneously the natural game theoretical requirements of Core Compatibility and Strong Monotonicity. To obtain the result we characterize the Shapley value on the class of totally balanced games and also on the class of exact games as being the only risk allocation method satisfying Strong Monotonicity, Equal Treatment Property and Efficiency. Moreover, we clarify and interpret the related game theoretical requirements that have appeared in the literature so far and have been applied to risk allocation.

Keywords: Coherent Measures of Risk, Risk Allocation Games, Totally Balanced Games, Exact Games, Shapley value, Core

JEL Classification: C71, G10
\end{abstract}

${ }^{*}$ We would like to thank two anonymous referees, Edina Berlinger, Ferenc Forgó, P. Jean-Jacques Herings, László Á. Kóczy, Tamás Solymosi and participants of the 4th World Congress of the Game Theory Society and SING7 for helpful comments.

†Department of Finance, Corvinus University of Budapest and "Momentum" Game Theory Research Group, Centre for Economic and Regional Studies, Hungarian Academy of Sciences. E-mail: peter.csoka@uni-corvinus.hu. Péter Csóka thanks funding from HAS (LP-004/2010) and OTKA (PD 105859).

${ }^{\ddagger}$ Corresponding author: Department of Mathematics, Corvinus University of Budapest, MTA-BCE "Lendület" Strategic Interactions Research Group and Faculty of Business and Economics, University of Pécs, 1093 Hungary, Budapest, Fővám tér 13-15. E-mail: miklos.pinter@uni-corvinus.hu. Miklós Pintér acknowledges the support by SPOR 4.2.2.B-15/1/konv, the János Bolyai Research Scholarship of the Hungarian Academy of Sciences and OTKA (K 101224). 


\section{Introduction}

If a firm (financial enterprise, bank, insurance company, investment fund, portfolio, etc.) consists of divisions (individuals, products, subportfolios, risk factors, etc.), not only is it important to measure properly the risk of the firm, but also to allocate the risk capital of the firm to the divisions. From now on we refer to this problem as risk allocation problem and use the terms firm and divisions while keeping all the possible applications in mind.

In terms of measuring risk, we would like to model Expected Shortfall Acerbi and Tasche, 2002), which is promoted by the Basel Committee on Banking Supervision (BCoBS, 2014) as the future industry standard, replacing Value-at-Risk. In this setting, a measure of risk assigns a real number to the future profit and loss random variable from the perspective of present. In order to keep the paper as simple as possible and to reach readers who are not familiar with the advanced mathematics of finance, we work with a finite probability space, where the portfolios of divisions are represented by realization vectors. We emphasize that assuming a finite probability space is not an infringement of generality, all of our results hold when the portfolios are described by bounded random variables on a measure space. A coherent measure of risk (Artzner et al, 1999) satisfies four natural properties (see Definition 2.1). A prominent example is the $k$-Expected Shortfall (Acerbi and Tasche, 2002), which is the average of the worst $100 k$ percent of the losses for $0 \leq k \leq 1$. On the other hand, Value-at-Risk is not a coherent measure of risk (Artzner et al, 1999).

When using a coherent measure of risk, the risk of the portfolio of the firm is at most as much as the sum of the risks of the portfolios of the divisions. Thus there is usually a diversification benefit, which should be allocated somehow. Risk (capital) allocation games (Denault, 2001) are transferable utility (TU) cooperative games defined to model such risk allocation. In a TU game using the values (the negative of the risk) of the coalitions (subsets) of the players (divisions) a solution concept (a risk allocation rule) determines how to share the value of the grand coalition (the firm). An allocation is in the core if the total value of the grand coalition is allocated (Efficiency) in such a way that no coalition of the players fairs better by acting alone. A totally balanced game has a non-empty core in each of its subgames, where a subgame is obtained by considering only a subset of the players.

Csóka et al (2009) showed that the class of risk allocation games (using coherent measures of risk) coincides with the class of totally balanced games. That is, on the one hand, for any risk allocation game there is a core allocation, a stable way to allocate risk using an allocation rule satisfying Core Compatibility $(C C)$. On the other hand, any totally balanced game can be given by a properly chosen risk allocation game. Csóka and Herings (2014) defined risk allocation games when divisions have illiquid portfolios and show that the class of risk allocation games with liquidity also coincides with the class of totally bal- 
anced games. Csóka et al (2009) also proved that the class of risk allocation games with no aggregate uncertainty equals the class of exact games (Schmeidler, 1972), where for each coalition there is a core allocation allocating the stand-alone value of the coalition.

In addition to $\mathrm{CC}$, in this paper we consider two further fairness properties of risk capital allocation rules: Equal Treatment Property (ETP) and Strong Monotonicity (SM). The motivation comes from Young]s axiomatization (Young, 1985) of the Shapley value (Shapley, 1953), where he showed that on the class of all games the Shapley value is the only solution concept satisfying Efficiency, ETP and SM. It is well-known that the Shapley value does not satisfy $\mathrm{CC}$ in general, hence the properties of CC, ETP and SM cannot be satisfied simultaneously on the class of all games.

However, the validity of an axiomatization of a solution can vary from subclass to subclass, e.g. Shapley]s axiomatization of the Shapley value (see Section 4) is valid on the class of monotone games but not valid on the class of strictly monotone games. In the case of risk allocation games (in particular totally balanced games or exact games) we generalize Young s result, and show that his axiomatization remains valid on the classes of totally balanced and exact games. We will prove (Theorem 3.4 that on the class of risk allocation games there is no risk allocation rule satisfying CC, ETP and SM at the same time.

We also interpret the requirements in the risk allocation setting as follows. $\mathrm{CC}$ is satisfied if all the risk of the firm is allocated in such a way that no group of the divisions can improve by allocating only the risk of the group, the risk allocation can rightly be seen stable. The blocking interpretations of the core can be questioned by saying that divisions cannot really leave the firm, see also Homburg and Scherpereel (2008). However, CC can also be viewed in an other way. The allocated risk to each coalition should be at least as much as the risk increment the coalition causes by joining the complementary coalition. ETP ensures that if two divisions have the same stand-alone risk and also they contribute the same risk to all the subsets of divisions not containing them, then they are treated equally, that is, the same risk capital is allocated to them. In other words, ETP states that if two divisions are not distinguishable from the point of view of risk, then they must be evaluated equally. SM requires that if a division weakly reduces its stand-alone risk and also its risk contribution to all the subsets of the other divisions (hence weakly increases its relative performance), then its allocated capital should not increase. Therefore, SM is closely related to Incentive Compatibility.

We will also prove (Proposition 3.5) that on the class of totally balanced (exact) games if a risk allocation rule meets $\mathrm{CC}$ and $\mathrm{SM}$ together, then there does exist a risk allocation rule which satisfies CC, SM and ETP together. However, we know from Theorem 3.4 that these three properties cannot hold at the same time, hence we find two (independent) 
requirements to blame for the impossibility result: CC and SM, one has to give up at least one of them.

Finally, in Example 3.8 we will illustrate that our impossibility result (Theorem 3.7) can be made stronger, because in all practical applications SM can be replaced by the following requirement: if a division's stand-alone performance increases, then its allocated risk should not increase. That is, in practice there does not exist a risk allocation rule which satisfies CC and Incentive Compatibility at the same time.

The paper is organized as follows. In the following section we introduce the notations and notions for transferable utility cooperative games in general and risk allocations games in particular. In Section 3 we present our impossibility result. In Section 4 we show how our impossibility result is related to other results in the literature. The last section concludes.

\section{Risk allocation games}

We will use the following notations and notions: $|N|$ is for the cardinality of a finite set $N$ and $2^{N}$ is the power set of $N$.

Let $N$ denote the finite set of players. A cooperative game with transferable utility (game, for short) is a function $v: 2^{N} \rightarrow \mathbb{R}$ such that $v(\emptyset)=0$. The class of games with player set $N$ is denoted by $\mathcal{G}^{N}$. For a game $v \in \mathcal{G}^{N}$ and a coalition $C \in 2^{N}$, a subgame $v_{C}$ is obtained by restricting $v$ to the subsets of $C$.

An allocation is a vector $x \in \mathbb{R}^{N}$, where $x_{i}$ is the payoff of player $i \in N$. An allocation $x$ yields payoff $x(C)=\sum_{i \in C} x_{i}$ to a coalition $C \in 2^{N}$. An allocation $x \in \mathbb{R}^{N}$ is called Efficient, if $x(N)=v(N)$ and Coalitionally Rational if $x(C) \geq v(C)$ for all $C \in 2^{N}$. The core (Gillies, 1959) is the set of Efficient and Coalitionally Rational allocations. The core of game $v$ is denoted by core $(v)$.

Let $v \in \mathcal{G}^{N}$ and $i \in N$ be a game and a player, and for all $C \subseteq N$ let $v_{i}^{\prime}(C)=$ $v(C \cup\{i\})-v(C)$ denote player $i$ 's marginal contribution to coalition $C$ in game $v$. Then $v_{i}^{\prime}$ is called player $i$ 's marginal contribution function in game $v$. Moreover, players $i$ and $j$ are equivalent in game $v, i \sim^{v} j$, if for every $C \subseteq N$ such that $i, j \notin C$ we have that $v_{i}^{\prime}(C)=v_{j}^{\prime}(C)$.

A game is totally balanced, if each of its subgame has a non-empty core. Let $\mathcal{G}_{\mathrm{tb}}^{N}$ denote the class of totally balanced games with player set $N$.

An interesting subclass of totally balanced games is the class of exact games (Schmeidler, 1972). A game $v$ is exact, if for each coalition $C$ there exists an allocation $x \in$ core $(v)$ such that $x(C)=v(C)$. Let $\mathcal{G}_{\mathrm{e}}^{N}$ denote the class of exact games with player set $N$.

Throughout the paper we consider single-valued solutions. The function $\psi: A \rightarrow \mathbb{R}^{N}$, defined on $A \subseteq \mathcal{G}^{N}$, is called solution on the class of games $A$. In the context of risk 
allocation, we refer to solutions as risk allocation rules.

For any game $v \in \mathcal{G}^{N}$ the Shapley solution $\phi$ is given by

$$
\phi_{i}(v)=\sum_{C \subseteq N \backslash\{i\}} v_{i}^{\prime}(C) \frac{|C| !(|N \backslash C|-1) !}{|N| !} \quad i \in N
$$

where $\phi_{i}(v)$ is also called the Shapley value (Shapley, 1953) of player $i$ in game $v$.

To define risk allocation games we use the setup of Csóka et al (2009). Let $S$ denote the finite number of states of nature and consider the set $\mathbb{R}^{S}$ of realization vectors. State of nature $s$ occurs with probability $p_{s}>0$, where $\sum_{s=1}^{S} p_{s}=1$. The vector $X \in \mathbb{R}^{S}$ represents a division's possible profit and loss realizations. The amount $X_{s}$ is the division's payoff in state of nature $s$. Negative values of $X_{s}$ correspond to losses.

A measure of risk is a function $\rho: \mathbb{R}^{S} \rightarrow \mathbb{R}$ measuring the risk of a realization vector.

Definition 2.1. A function $\rho: \mathbb{R}^{S} \rightarrow \mathbb{R}$ is called a coherent measure of risk Artzner et al, 1999) if it satisfies the following axioms:

1. Monotonicity: for all $X, Y \in \mathbb{R}^{S}$ such that $Y \geq X$, we have $\rho(Y) \leq \rho(X)$.

2. Subadditivity: for all $X, Y \in \mathbb{R}^{S}$, we have $\rho(X+Y) \leq \rho(X)+\rho(Y)$.

3. Positive homogeneity: for all $X \in \mathbb{R}^{S}$ and $h \in \mathbb{R}_{+}$, we have $\rho(h X)=h \rho(X)$.

4. Translation invariance: for all $X \in \mathbb{R}^{S}$ and $a \in \mathbb{R}$, we have $\rho\left(X+a 1^{S}\right)=\rho(X)-a$.

For an interpretation of the axioms see Acerbi and Scandolo (2008), who justify them for incorporating liquidity risk as well. A prominent example for a coherent measure of risk is the $k$-Expected Shortfall (Acerbi and Tasche, 2002), which is the average of the worst $100 k$ percent of the losses for $0 \leq k \leq 1$. Note that for $k=0$ we get the maximal loss, which is then also a coherent measure of risk.

Let the matrix of realization vectors corresponding to the divisions be given by $X \in$ $\mathbb{R}^{S \times N}$, and let $X_{\cdot i}$ denote column $i$ of $X$, the realization vector of division $i$. For a coalition of divisions $C \in 2^{N}$, let $X_{C}=\sum_{i \in C} X_{\cdot i}$.

A risk environment is a tuple $(N, S, p, X, \rho)$, where $N$ is the set of divisions, $S$ indicates the number of states of nature, $p=\left(p_{1}, \ldots, p_{S}\right)$ is the vector of realization probabilities of the various states, $X$ is the matrix of realization vectors, and $\rho$ is a coherent measure of risk.

A risk allocation game assigns to each coalition of divisions the negative of the risk the coalition runs in its aggregate portfolio. 
Definition 2.2. Given the risk environment $(N, S, p, X, \rho)$, a risk allocation game is a game $v \in \mathcal{G}^{N}$, where

$$
v(C)=-\rho\left(X_{C}\right) \text { for all } C \in 2^{N} \backslash\{\emptyset\},
$$

and $v(\emptyset)=0$.

Let $\mathcal{G}_{\mathrm{r}}^{N}$ denote the family of risk allocation games with player set $N$. In such games, according to Equation (1), the larger the risk of any subset of divisions, the lower its value. We illustrate the definition of the risk allocation game by the following example.

Example 2.3. Consider the following risk environment $(N, S, p, X, \rho)$. We have 3 divisions, 7 states of nature with equal probability of occurrence. Risk is calculated by using the matrix of realization vectors in the first three columns of Table 1 and the maximum loss risk measure.

\begin{tabular}{c|ccc|cccc}
$S \backslash X_{C}$ & $X_{\{1\}}$ & $X_{\{2\}}$ & $X_{\{3\}}$ & $X_{\{1,2\}}$ & $X_{\{1,3\}}$ & $X_{\{2,3\}}$ & $X_{\{1,2,3\}}$ \\
\hline 1 & 0 & 1 & 1 & 1 & 1 & 2 & 2 \\
2 & 1 & 0 & 1 & 1 & 2 & 1 & 2 \\
3 & 1 & 1 & 0 & 2 & 1 & 1 & 2 \\
4 & $1 / 2$ & $1 / 2$ & 1 & 1 & $3 / 2$ & $3 / 2$ & 2 \\
5 & 0 & 1 & 0 & 1 & 0 & 1 & 1 \\
6 & 1 & $1 / 2$ & $1 / 2$ & $3 / 2$ & $3 / 2$ & 1 & 2 \\
7 & 0 & 1 & 0 & 1 & 0 & 1 & 1 \\
\hline$\rho(C)$ & 0 & 0 & 0 & -1 & 0 & -1 & -1 \\
\hline$v(C)$ & 0 & 0 & 0 & 1 & 0 & 1 & 1
\end{tabular}

Table 1: The matrix of realization vectors of a risk environment and the resulting totally balanced risk allocation game $v$ using the maximum loss as a measure of risk.

Note that for all $C \in 2^{N} \backslash\{\emptyset\}$ the value function is given by $v(C)=-\rho\left(X_{C}\right)=$ $\min _{s \in S} X_{C, s}$.

If the rows of a matrix of realization vectors sum up to the same number, then there is no aggregate uncertainty. Formally: a matrix of realization vectors $X \in \mathbb{R}^{S \times N}$ has no aggregate uncertainty, if there exists a number $\alpha \in \mathbb{R}$ such that $X_{N}=\alpha 1^{S}$. Let $\mathcal{G}_{\text {rnau }}^{N}$ denote the family of risk allocation games with no aggregate uncertainty and with player set $N$. 
Theorem 2.4. (Csóka et al, 2009; Csóka and Herings, 2014) The class of risk allocation games with or without liquidity coincides with the class of totally balanced games, that is, $\mathcal{G}_{\mathrm{r}}^{N}=\mathcal{G}_{\mathrm{tb}}^{N}$. Moreover, the class of risk allocation games with no aggregate uncertainty equals the class of exact games, that is, $\mathcal{G}_{\text {rnau }}^{N}=\mathcal{G}_{\mathrm{e}}^{N}$.

Note that by Theorem 2.4 all risk allocation games with or without liquidity are totally balanced (also the one in Example 2.3), and if there is no aggregate uncertainty, then all of them are exact. Moreover, for any totally balanced (exact) game there is a risk environment including a coherent measure of risk (with no aggregate uncertainty) that generates the game using Equation (1).

A risk allocation rule shows how to share the risk of the firm among the divisions. Since the situation can be converted into a TU game, a risk allocation rule can also be a solution in cooperative game theory, such as e.g. the Shapley solution. We illustrate the marginal contribution function and the fact that the Shapley solution is not always in the core by continuing Example 2.3 .

Example 2.5. Consider the risk allocation game in Example 2.3.

\begin{tabular}{c|c|ccc|ccc|c}
$C$ & $\emptyset$ & $\{1\}$ & $\{2\}$ & $\{3\}$ & $\{1,2\}$ & $\{1,3\}$ & $\{2,3\}$ & $\{1,2,3\}$ \\
\hline$v(C)$ & 0 & 0 & 0 & 0 & 1 & 0 & 1 & 1 \\
$v_{1}^{\prime}(C)$ & 0 & 0 & 1 & 0 & 0 & 0 & 0 & \\
$v_{2}^{\prime}(C)$ & 0 & 1 & 0 & 1 & 0 & 1 & 0 & \\
$v_{3}^{\prime}(C)$ & 0 & 0 & 1 & 0 & 0 & 0 & 0 &
\end{tabular}

Table 2: The marginal contribution functions of players 1, 2 and 3.

Note that player 2 has a higher marginal contribution than the others, which is also expressed by the Shapley value, since $\phi(v)=(1 / 6,2 / 3,1 / 6)$. However, coalition $\{1,2\}$ is a blocking coalition for the Shapley solution in game $v$, since $v(\{1,2\})=1>5 / 6=1 / 6+2 / 3$, that is, $\phi(v) \notin$ core $(v)$.

\section{The impossibility result}

Next, we introduce the four basic properties (axioms) that we focus on.

Definition 3.1. The solution $\psi$ on subclass $A \subseteq \mathcal{G}^{N}$ satisfies

- Core Compatibility $(C C)$ if for each $v \in A$, we have $\psi(v) \in$ core $(v)$,

- Efficiency if for each $v \in A$, we have $\sum_{i \in N} \psi_{i}(v)=v(N)$, 
- Equal Treatment Property (ETP) if for all $v \in A$ and $i, j \in N$ such that $i \sim^{v} j$, we have $\psi_{i}(v)=\psi_{j}(v)$,

- Strong Monotonicity (SM) if for all $v, w \in A$ and $i \in N$ such that $v_{i}^{\prime} \leq w_{i}^{\prime}$, we have $\psi_{i}(v) \leq \psi_{i}(w)$.

The financial interpretations of the requirements are as follows. CC is satisfied if the risk allocation rule results in a core allocation, that is, all the risk of the firm is allocated in such a way that no group of the divisions can fair better by allocating only the risk of the group. A core compatible risk allocation can rightly be viewed stable. Notice that for a Coalitionally Rational allocation $x$ we have that $x(N \backslash C) \geq v(N \backslash C)$ for all $C \in 2^{N}$, which, together with Efficiency imply that $x(C) \leq v(N)-v(N \backslash C)$ for all $C \in 2^{N}$. That is, in a core allocation the value ("diversification gain") allocated to each coalition can be at most as much as its contribution to the complementary coalition. To put it differently, the allocated risk to each coalition should be at least as much as the risk increment the coalition causes by joining the complementary coalition.

Efficiency is implied by $\mathrm{CC}$, since it requires that all the risk of the firm should be allocated to the divisions.

ETP makes sure that if two divisions have the same stand-alone risk and also they contribute the same risk to all the subsets of divisions not containing them, then they are treated equally, that is, the same risk capital is allocated to them. In other words, if two divisions are the same from the viewpoint of risk, then by ETP the same risk is assigned to both.

SM requires that if a division weakly reduces its stand-alone risk and also its risk contribution to all the subsets of the other divisions, hence weakly increases its relative performance, then its allocated capital should not increase. Thus as a kind of Incentive Compatibility notion, weakly better relative performance is weakly rewarded. Note that it follows from SM that for any $v, w \in A, i \in N$ such that $v_{i}^{\prime}=w_{i}^{\prime}$, we have $\psi_{i}(v)=\psi_{i}(w)$ (called Marginality).

Theorem 3.2. (Theorem 2, Young (1985)). Let $\psi$ be a solution on the class of all games. Then solution $\psi$ satisfies Efficiency, ETP and SM (Marginality) if and only if $\psi=\phi$, that is, if and only if it is the Shapley solution.

Note that risk allocation games form a proper subset of all games, since they are always totally balanced. In the following, we prove a Theorem 3.2 type result on the classes of totally balanced and exact games.

Theorem 3.3. Let $\psi$ be a solution on the class of totally balanced (exact) games. Then solution $\psi$ satisfies Efficiency, ETP and SM (Marginality) if and only if $\psi=\phi$, that is, if and only if it is the Shapley solution. 
Proof. If: See e.g. Young (1985).

Only if: Let $u_{T}$ denote the unanimity game on coalition $T$, that is, for all $C \subseteq N$ : $u_{T}(C)=\left\{\begin{array}{ll}1, & \text { if } C \supseteq T \\ 0 & \text { otherwise }\end{array}\right.$.

Next, we generalize Theorem 3.2 for such classes of games where if a game $v$ is in the class, then for all $\alpha>0$ and coalition $T$ we have that $v+\alpha u_{T}$ is also in the class. Notice that the class of totally balanced (exact) games is such class of games, since we get the required core allocations by distributing $\alpha$ among the members of coalition $T$ in a proper way.

Let $v$ be a totally balanced (exact) game and let us decompose it to the unique sum of unanimity games such as $v=\sum_{T \subseteq N} \alpha_{T} u_{T}$. Moreover, let $\alpha^{m}=\max _{T \subseteq N} \alpha_{T}, v^{*}=$ $\alpha^{m} \sum_{T \subseteq N} u_{T}$, and $v_{d}=v^{*}-v$.

Notice that $v^{*}$ is a totally balanced (exact) game and in $v_{d}=\sum_{T \subseteq N} \beta_{T} u_{T}$ we have that $\beta_{T} \geq 0$ for all $T \subseteq N$. For each game $w$ define the index $I(w)$ as follows: $I(w)=\mid\left\{\gamma_{T} \neq\right.$ $\left.0: w=\sum_{T \subseteq N} \gamma_{T} u_{T}\right\} \mid$.

The proof goes by induction on $I\left(v_{d}\right)$.

If $I\left(v_{d}\right)=0$, then $v=v^{*}$, all players are equivalent in game $v$, so Efficiency and ETP imply that solution $\psi$ is well-defined (unique) for game $v$.

Let $k$ be an integer such that $0<k<2^{|N|}-1$. Assume that for each totally balanced (exact) game $w$ such that $I\left(w_{d}\right) \leq k, \psi(w)$ is well-defined. Then, let $v$ be a totally balanced (exact) game such that $I\left(v_{d}\right)=k+1$. Consider the decomposition $v_{d}=\sum_{T \subseteq N} \beta_{T} u_{T}$, that is, $v=v^{*}-\sum_{T \subseteq N} \beta_{T} u_{T}$, where $\beta_{T} \geq 0$ for all $T \subseteq N$.

First, if it exists, take any player $i$ for which there exists $T \subseteq N, \beta_{T}>0$ such that $i \notin T$. Let $v^{k}=v+\beta_{T} u_{T}$. Notice that since $\beta_{T}>0$ we have that $v^{k}$ is totally balanced (exact). Moreover, $I\left(v_{d}^{k}\right)=k$ and by the induction hypothesis $\psi\left(v^{k}\right)$ is well-defined. Since $v_{i}^{\prime}=\left(v+\beta_{T} u_{T}\right)_{i}^{\prime}$, SM (Marginality) implies that $\psi_{i}(v)=\psi_{i}\left(v^{k}\right)$.

Second, if they exist, take the remaining players, that is, take all $i$ such that $i \in T \subseteq N$ for all $T \subseteq N$ where $\beta_{T}>0$. These players are equivalent in $v$ (since they are equivalent in all games $\beta_{T} u_{T}, \beta_{T}>0$ ), so by ETP they get the same value.

Finally, by Efficiency, solution $\psi$ is well-defined for game $v$.

Since the Shapley solution satisfies Efficiency, ETP and SM (Marginality) and $\psi$ is well-defined (unique) using these properties, we have $\psi=\phi$.

Using Theorem 3.3 we can state the following.

Theorem 3.4. There is no risk allocation rule meeting the properties of CC, ETP and SM (Marginality) at the same time. 
Proof. Theorem 3.3 is characterizing the Shapley value as being the only risk allocation rule satisfying Efficiency, ETP and SM (Marginality) on the class of risk allocation games with or without liquidity (with no aggregate uncertainty), which, by Theorem 2.4 coincides with the class of totally balanced (exact) games. By Example 2.5 the Shapley value is not always in the core for totally balanced games. Moreover, Rabie (1981) showed by an example that for at least five players the Shapley value is not in the core for exact games.

So far we have established that the Shapley value is the only risk allocation rule satisfying Efficiency, ETP and SM (Marginality) on the class of risk allocation games with or without liquidity (with or without aggregate uncertainty), but it does not satisfy CC in general, hence there is no hope to satisfy CC, ETP and SM (Marginality) at the same time. Next, we show that Theorem 3.4 can be refined using the following proposition.

Proposition 3.5. Suppose that $\psi$ is a solution satisfying CC and SM on the class of totally balanced (exact) games. Then there exists a solution $\bar{\psi}$ on the class of totally balanced (exact) games which satisfies CC, SM and also meets ETP.

Proof. For all $v \in \mathcal{G}_{t b}^{N}\left(v \in \mathcal{G}_{e}^{N}\right)$ and player $i \in N$ let

$$
\bar{\psi}(v)_{i}=\frac{1}{|\Pi(N)|} \sum_{\pi \in \Pi(N)} \psi(v \circ \pi)_{\pi(i)}
$$

where $\Pi(N)$ is the class of permutations on set $N$.

Then it is easy to see that for each $\pi \in \Pi(N)$ game $v \circ \pi$ is a totally balanced (exact) game, and $v_{i}^{\prime} \geq w_{i}^{\prime}$ if and only if $(v \circ \pi)_{\pi(i)}^{\prime} \geq(w \circ \pi)_{\pi(i)}^{\prime}$. Therefore $\bar{\psi}$ also meets CC and SM.

Take a game $v \in \mathcal{G}_{t b}^{N}\left(v \in \mathcal{G}_{e}^{N}\right)$ such that players $i$ and $j$ are equivalent in game $v$. Let $\pi_{i j} \in \Pi(N)$ be the permutation such that $\pi_{i j}(k)=k$ if $k \in N \backslash\{i, j\}$ and $\pi_{i j}(i)=j$. Then for each permutation $\pi \in \Pi(N)$ we have that $v \circ \pi \circ \pi_{i j}=v \circ \pi$. Therefore $\sum_{\pi \in \Pi(N)} \psi(v \circ \pi)_{\pi(i)}=\sum_{\pi \in \Pi(N)} \psi(v \circ \pi)_{\pi(j)}$.

Thus ETP is not independent from CC and SM on the class of totally balanced (exact) games.

Remark 3.6. Notice that Proposition 3.5 can be (straightforwardly) generalized to the case where CC is replaced by Efficiency, or SM is replaced either by Marginality or by Coalitional Monotonicity (see Young (1985)). Moreover, the domain of axiomatization can be any class of games which is closed under permutations. The relationship among the above mentioned concepts of monotonicity and CC was also examined by Young (1985) and Housman and Clark (1998). 
Using Proposition 3.5 we have the following theorem.

Theorem 3.7. There is no risk allocation rule satisfying the requirements of $C C$ and $S M$ (Marginality) at the same time.

Proof. Assume that there is a risk allocation rule meeting the requirements of $\mathrm{CC}$ and SM (Marginality) at the same time. Then by Proposition 3.5 there exists a solution $\bar{\psi}$ on the class of totally balanced (exact) games which satisfies CC, SM and also satisfies ETP, contradicting Theorem 3.4 .

The following example demonstrates that in practice SM can be replaced by the following requirement: if a divisions' stand-alone (reported) performance increases, then its allocated risk should not increase. That is, in practice SM can be replaced by Incentive Compatibility.

Example 3.8. Consider the risk environments $(N, S, p, X, \rho)$ and $(N, S, p, Y, \rho)$, where $S=\left\{s_{1}, \ldots, s_{19}\right\}, N=\{1,2,3,4,5\}, p$ is the uniform distribution, $\rho$ is the maximal loss risk measure, and $X$ is as in Table 3, $Y$ is the same as $X$ except for division 2, where at state $s_{19}$ it performs worse or reports worse performance by 3 , that is, $Y_{\{2\}}=$ $X_{\{2\}}-(0,0,0,0,0,0,0,0,0,0,0,0,0,0,0,0,0,0,3)^{\top}$.

Let the risk allocation games $w$ and $v$ be generated by risk environments $(N, S, p, X, \rho)$ and $(N, S, p, Y, \rho)$, respectively. It is easy to see that we get the games by Young (1985) (Theorem 1, pp. 69), where $\operatorname{core}(w)=(3,0,0,6,3)$ and core $(v)=(0,1,2,7,1)$. Moreover, $Y_{\{2\}}<X_{\{2\}}$, thus in this case division 2 can outsmart any risk allocation method satisfying CC by performing worse or reporting less profit (like in $Y$ ), because then it gets 1 instead of 0. In other words there is no risk allocation rule satisfying CC and Incentive Compatibility.

\section{Relations to other axiomatic approaches}

Other axiomatic approaches in the literature related to our work will be discussed below.

\subsection{Denault (2001)}

Denault (2001) considers (a version of) Shapleyls axiomatization of the Shapley value (Shapley, 1953), and concludes that there is no relevant risk allocation rule which satisfies CC, Symmetry, Dummy Player Property and Additivity.

By Symmetry Denault (2001) means ETP, which is a bit misleading, since Symmetry means something different: A solution $\psi$ meets Symmetry if every game $v \in \mathcal{G}^{N}$ and permutation $\pi$ on $N$ such that $v=v \circ \pi$, we have that for each player $i \in N, \psi_{i}(v)=\psi_{\pi(i)}(v)$ (see Peleg and Sudhölter (2007)). 


\begin{tabular}{c|ccccc} 
& $X_{\{1\}}$ & $X_{\{2\}}$ & $X_{\{3\}}$ & $X_{\{4\}}$ & $X_{\{5\}}$ \\
\hline$s_{1}$ & 0 & 100 & 0 & 100 & 100 \\
$s_{2}$ & 100 & 100 & $3 / 2$ & 100 & $3 / 2$ \\
$s_{3}$ & 1 & 1 & 1 & 100 & 100 \\
$s_{4}$ & 0 & 0 & 100 & 0 & 100 \\
$s_{5}$ & 0 & 0 & 100 & 100 & 0 \\
$s_{6}$ & 3 & 100 & 3 & 3 & 100 \\
$s_{7}$ & 0 & 100 & $3 / 2$ & 100 & $3 / 2$ \\
$s_{8}$ & 0 & 100 & 100 & 0 & 0 \\
$s_{9}$ & 100 & 0 & 0 & 0 & 100 \\
$s_{10}$ & 100 & 0 & $3 / 2$ & 100 & $3 / 2$ \\
$s_{11}$ & 100 & 3 & 100 & 3 & 3 \\
$s_{12}$ & 100 & 100 & $3 / 2$ & 0 & $3 / 2$ \\
$s_{13}$ & 3 & 0 & 3 & 3 & 100 \\
$s_{14}$ & 0 & 0 & 3 & 100 & 0 \\
$s_{15}$ & 2 & 5 & 100 & 3 & 2 \\
$s_{16}$ & 3 & 100 & 3 & 3 & 0 \\
$s_{17}$ & 100 & 3 & 0 & 3 & 3 \\
$s_{18}$ & 3 & 0 & 0 & 6 & 3 \\
$s_{19}$ & 0 & 4 & 2 & 7 & 1
\end{tabular}

Table 3: The matrix $X$ in Example 3.8 
It is worth noticing that the axioms ETP and Symmetry are not related to each other in general, but for totally balanced games Symmetry is stronger than ETP.

Furthermore, a solution $\psi$ satisfies the Dummy Player Property if every game $v \in \mathcal{G}^{N}$ and player $i \in N$ such that $v_{i}^{\prime}(S)=c_{i}$ for all $S \subseteq N \backslash\{i\}$, we have $\psi_{i}(v)=c_{i}$. This axiom is stronger than the related Shapley]'s axiom (see Axiom 2 and Lemma 1 in Shapley (1953)).

A solution $\psi$ meets Additivity if for all games $v, w \in \mathcal{G}^{N}$ we have $\psi(v+w)=\psi(v)+\psi(w)$. Considering risk allocation games it is very difficult to interpret this axiom (there is a similar problem in regression games, for more details see Pintér (2011)). The problem is that while it is reasonable to add up the matrices of realization vectors of two risk allocation games, the risk allocation game generated by the sum of two risk environments is typically not the sum of the two risk allocation games generated by the two risk environments. Actually, Additivity (on environments) only has a bite if the portfolios for each coalition in the games to be added are comonotonic, that is, they are a positive function of the same random variable. Therefore, in our opinion Shapley]s axiomatization of the Shapley value does not fit with the risk allocation problem.

Moreover, Denault (2001) does not prove that Shapley]s axiomatization is true on the class of risk allocation games. This is a problem because even if an axiomatization holds on the whole class of games it does not mean that the axiomatization holds on a subclass of games. E.g. Shapley's axiomatization does not hold on the class of strictly monotone games, where e.g. the egalitarian solution, where the value of the grand coalition is divided equally among the players, also satisfies Shapley]s axioms. To the best of our knowledge the problem of whether Shapley]s axioms characterize the Shapley value on the class of risk allocation games is open.

What is also problematic is that Denault (2001) is imposing very strict necessary conditions for the Shapley value to be in the core, which are not sufficient. In fact, we are not aware of any necessary and sufficient conditions for the Shapley value to be in the core.

To sum up, Denault's conclusion on a proper risk allocation method is only partially verified, so it is worth to examine the problem further.

\subsection{Other related papers}

Drehmann and Tarashev (2013) also consider Shapley's axiomatization of the Shapley value for systemic risk allocation games but it has the same shortcomings as Denault (2001) (the axiomatization is not proved and additivity is not so natural to require) and they even interpret Additivity as Efficiency.

Valdez and Chernih (2003) showed that for elliptically contoured distributions the covariance (or beta) method satisfies CC (No Undercut), ETP (the authors call it Symmetry) and Consistency (requiring that the allocation method should be independent of the hier- 
archical structure of the firm). However, Kim and Hardy $(2009)$ showed that it is not even true that the covariance method satisfies Core Compatibility in this setting. Moreover, profit and loss distributions of financial assets are not elliptically contoured, but heavy tailed (see for instance Cont (2001)), hence our approach is clearly more relevant by not restricting the probability distributions.

Kalkbrener (2005) showed that Linear Aggregation, Diversification and Continuity characterizes the gradient principle (or Euler method, where risk is allocated as a result of slightly increasing the weights of the divisions) to be the only allocation which satisfies these requirements. Although those requirements are also natural, they are not related to the properties of CC, ETP or SM. In fact Kalkbrener (2005) explicitly assumed that the risk allocated to a division does not depend on the decomposition of the other divisions, only on the firm itself, which is a strange and strong assumption.

Finally, there is a related impossibility result by Buch and Dorfleitner (2008). They showed that if one uses the gradient principle to allocate risk and ETP (the authors call it Symmetry) is satisfied, then the measure of risk must be linear, not allowing for any diversification benefits. In other words, Buch and Dorfleitner (2008) argue that ETP is to blame for the impossibility of fair risk allocation. In this paper, however, we are looking for a general risk allocation method, not the gradient one. Moreover, as our result (Theorem 3.7) indicates, in our setting it becomes clear that ETP cannot be blamed, but the fact that CC and SM exclude each other makes the impossibility result to hold.

\section{Conclusion}

We have shown that by using coherent measures of risk it is impossible to allocate risk satisfying simultaneously the natural requirements of Core Compatibility and Strong Monotonicity (in practice Incentive Compatibility). To obtain the result we have characterized the Shapley value on the class of totally balanced games and also on the class of exact games as being the only risk allocation method satisfying Strong Monotonicity, Equal Treatment Property and Efficiency. Both classes are proper subsets of all TU games, hence it is not obvious that the characterization by Young (1985) holds on them. We have also interpreted the axioms and our results in the risk allocation setting and clarified their relation to the existing literature.

Since due to the results in this paper the Shapley value is the only risk allocation method satisfying Strong Monotonicity, Equal Treatment Property and Efficiency, the deepness of our impossibility result can be captured by checking the necessary and sufficient condition for the Shapley value to be in the core. Since no such condition is known, one has to resort to simulation. Balog et al (2014) showed that our analytical result is not only a theoretical 
possibility. For randomly generated risk allocation games with 3 or 4 divisions the Shapley value is not in the core about 40-60\% of the cases on the average. Csóka (2015) reported only a few percent lower numbers for risk allocation games with liquidity. Moreover, for a higher number of divisions in general we expect higher numbers.

Therefore our result raises the practical problem: one has to give up one of Core Compatibility or Incentive Compatibility to allocate risk in practice too. Depending on the application at hand, the literature on cooperative games can help in deciding requirement to drop in light of the trade-off between theoretical versus computational gains and losses. For instance (as we have seen) the Shapley solution meets Efficiency, Equal Treatment Property and Strong Monotonicity (in practice Incentive Compatibility). Moreover, it is well-known that the nucleolus (Schmeidler, 1969) meets Core Compatibility and Equal Treatment Property, and there are many other well-analyzed solution concepts to choose from.

\section{References}

Acerbi C, Scandolo G (2008) Liquidity risk theory and coherent measures of risk. Quantitative Finance 8:681-692

Acerbi C, Tasche D (2002) On the Coherence of Expected Shortfall. Journal of Banking and Finance 26:1487-1504

Artzner PF, Delbaen F, Eber JM, Heath D (1999) Coherent Measures of Risk. Mathematical Finance 9:203-228

Balog D, Bátyi TL, Csóka P, Pintér M (2014) Properties of risk capital allocation methods: Core Compatibility, Equal Treatment Property and Strong Monotonicity, CEWP 13 $/ 2014$

BCoBS (2014) Consultative Document. Fundamental review of the trading book: outstanding issues. Basel Committee on Banking Supervision. Basel: Bank for International Settlements.

Buch A, Dorfleitner G (2008) Coherent risk measures, coherent capital allocation and the gradient allocation principle. Insurance: Mathematics and Economics 42(1):235-242

Cont R (2001) Empirical properties of asset returns: Stylized facts and statistical issues. Quantitative Finance 1:223-236 
Csóka P (2015) Fair risk allocation in illiquid markets. IEHAS Discussion Papers MT-DP2015/9, Institute of Economics, Centre for Economic and Regional Studies, Hungarian Academy of Sciences, 1-18

Csóka P, Herings JJ (2014) Risk allocation under liquidity considerations. Journal of Banking and Finance 49:1-9

Csóka P, Herings PJJ, Kóczy LÁ (2009) Stable Allocations of Risk. Games and Economic Behavior 67(1):266-276

Denault M (2001) Coherent Allocation of Risk Capital. Journal of Risk 4(1):1-34

Drehmann M, Tarashev N (2013) Measuring the systemic importance of interconnected banks. Journal of Financial Intermediation 22(4):586-607

Gillies DB (1959) Solutions to general non-zero-sum games, Contributions to the Theory of Games, vol IV. Princeton University Press

Homburg C, Scherpereel P (2008) How Should the Joint Capital be Allocated for Performance Measurement? European Journal of Operational Research 187(1):208-217

Housman D, Clark L (1998) Core and monotonic allocation methods. International Journal of Game Theory 27:611-616

Kalkbrener M (2005) An Axiomatic Approach to Capital Allocation. Mathematical Finance 15(3):425-437

Kim JHT, Hardy MR (2009) A capital allocation based on a solvency exchange option. Insurance: Mathematics and Economics 44(3):357-366

Peleg B, Sudhölter P (2007) Introduction to the theory of cooperative games, second edition edn. Kluwer

Pintér M (2011) Regression games. Annals of Operations Research 186(1):263-274

Rabie MA (1981) A Note on the Exact Games. International Journal of Game Theory 10(3-4):131-132

Schmeidler D (1969) The Nucleolus of a Characteristic Function Game. SIAM Journal on Applied Mathematics 17:1163-1170

Schmeidler D (1972) Cores of Exact Games. Journal of Mathematical Analysis and Applications 40:214-225 
Shapley LS (1953) A value for n-person games. In: Kuhn HW, Tucker AW (eds) Contributions to the Theory of Games II, Annals of Mathematics Studies, vol 28, Princeton University Press, Princeton, pp 307-317

Valdez E, Chernih A (2003) Wang's capital allocation formula for elliptically contoured distributions. Insurance: Mathematics and Economics 33:517-532

Young HP (1985) Monotonic Solutions of Cooperative Games. International Journal of Game Theory 14:65-72 